\title{
Contracting Alignment Patterns in the arrival directions of Ultra-High Energy Cosmic Rays induced by the Galactic Magnetic Field
}

\author{
Marcus Wirtz*, Martin Erdmann \\ III. Physikalisches Institut A, RWTH Aachen University, Aachen, Germany \\ E-mail: mwirtz@physik.rwth-aachen.de
}

\begin{abstract}
We update our method to search for the origins of ultra-high energy cosmic rays. First, we modify a model of the coherent component of the Galactic magnetic field by exploiting the existence of thread-like patterns in the arrival directions of ultra-high energy cosmic rays. This information is expected to complement our knowledge about the magnetic field by Faraday rotation and synchrotron emission measurements. Second, in a simultaneous fit to all cosmic rays considered we use this data-driven Galactic magnetic field model as a mass spectrometer and adapt the nuclear charges such that their extragalactic directions are concentrated in as few clusters as possible. During the fit, the nuclear charges are constrained by the individual energy and showerdepth measurements. We show in a simulated astrophysical scenario that source directions can be reconstructed even within a substantial isotropic background and in compliance with expected uncertainties of the knowledge of the Galactic magnetic field.
\end{abstract}

36th International Cosmic Ray Conference -ICRC2019-

July 24th - August 1st, 2019

Madison, WI, U.S.A.

\footnotetext{
*Speaker.
} 


\section{Introduction}

The research question of the origins of cosmic rays at the very highest energies observed so far is still unsolved. The discovery of a large-scale anisotropy in the form of a dipolar structure in the arrival directions of ultra-high energy cosmic rays (UHECRs) above $8 \mathrm{EeV} \mathrm{[1]} \mathrm{hints} \mathrm{that} \mathrm{UHECRs}$ have extragalactic origin since the dipole maximum is not correlating with the matter distribution of the Galaxy. Furthermore, an indication of a correlation was found with nearby starburst galaxies on intermediate angular scales at the $4 \sigma$ confidence level for energies above $39 \mathrm{EeV} \mathrm{[2].}$

In this energy range, cosmic rays are likely charged nuclei and thus deflected in cosmic magnetic fields, complicating the search for their sources. Presumably, the largest impact on the deflection arises from the magnetic field of the Milky Way. Despite numerous experimental efforts during the last decade $[3,4,5]$, the uncertainties in model predictions of the Galactic magnetic field (GMF) still remain substantial [6, 7]. The relation between the sources and the arrival directions on Earth is further complicated by the limited knowledge of the nuclear charges $Z$, which can be accessed only with poor sensitivity, e.g. through the maximum of the shower depth [8].

As a proposal to solve this challenging task, we present a method that first modifies a GMF model by including information of aligned patterns in the arrival directions as expected through deflections in the GMF. Second, in a simultaneous fit to the nuclear charges of the cosmic rays and their directions prior to their deflection in the GMF, we enforce them to originate in as few points as possible while keeping their charges compatible with their measured shower depths. While in a previous stage of this method [9] the GMF was assumed to be sufficiently known, in this work, we derive the above-mentioned correction of the model beforehand.

\section{Analysis strategy}

The aim of the presented method is to find a significant number of cosmic rays that can be assigned to point sources prior to deflections by the Galactic magnetic field.

For the high energy limit, the expected deflection for protons scales linearly with the inverse of their energy $E$ (cf. figure 1a). These so-called multiplet patterns were already searched for in data of the Pierre Auger Observatory [10], where no significant occurrence was found. However,

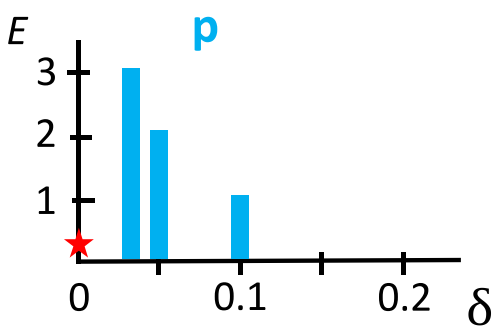

a)

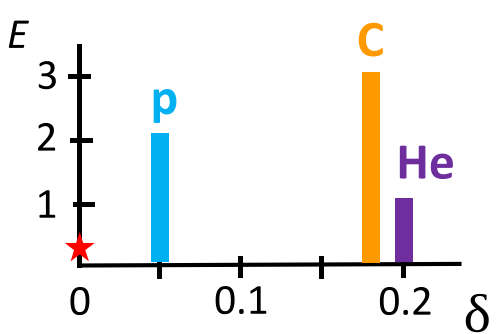

b)

Figure 1: One dimensional sketch of a) an energy dependent multiplet structure of protons with $1 / E$ deflection dependence and of b) a mixed composition multiplet of proton, helium and carbon with $Z / E$ deflection. The x-axis shows the deflection angle $\delta$ relative to their common source direction (red star) at $\delta=0$. 
as measurements reveal a mixed nuclear composition in cosmic-ray data at the highest energies [8], the absence of these patterns in data might be due to the additional scaling of the deflection with the nuclear charges $Z$ of the cosmic rays. Figure $1 \mathrm{~b}$ shows the same cosmic-ray energies than before, but this time with a mixed composition of protons $(Z=1)$, helium $(Z=2)$ and carbon $(Z=6)$ nuclei. An energy ordering of the pattern is not visible anymore due to the deflection angle scaling with the inverse rigidity $\delta \sim 1 / R=Z / E$. Clearly, a method which only considers the energies without accounting for the charges will not find the pattern anymore.

To overcome this issue, all nuclear charges of the cosmic rays and their origin before deflection are fitted at the same time. Due to the high dimensionality of the problem, we implement the deflection prescription in TensorFlow [11] and make use of its backpropagation technique.

\subsection{First stage: Modification of the Galactic magnetic field model}

In a first step, we modify the Galactic magnetic field model for the usage of contracting alignment patterns in the arrival directions of cosmic rays. Figure 2a shows arrival directions of cosmic rays with energies between $10^{18.5} \mathrm{eV}$ to $10^{20} \mathrm{eV}$ from a single hypothetical point source at galactic coordinates $l=0^{\circ}$ and $b=-45^{\circ}$ after deflections in the Galactic magnetic field models of Jansson \& Farrar (JF12) [3] and Pshirkov, Tinyakov, Kronberg and Newton-McGee (PT11-ASS) [4]. In the following, we will denote the spherical angle between the directions of deflection measured in the tangent plane to the celestial sphere as $\Psi$ (cf. figure 2a). As can be seen in figure $2 b$, the predictions of the two different Galactic magnetic field models differ substantially depending on the arrival direction and exhibit an overall median angle of $\Psi \approx 35^{\circ}[6]$.
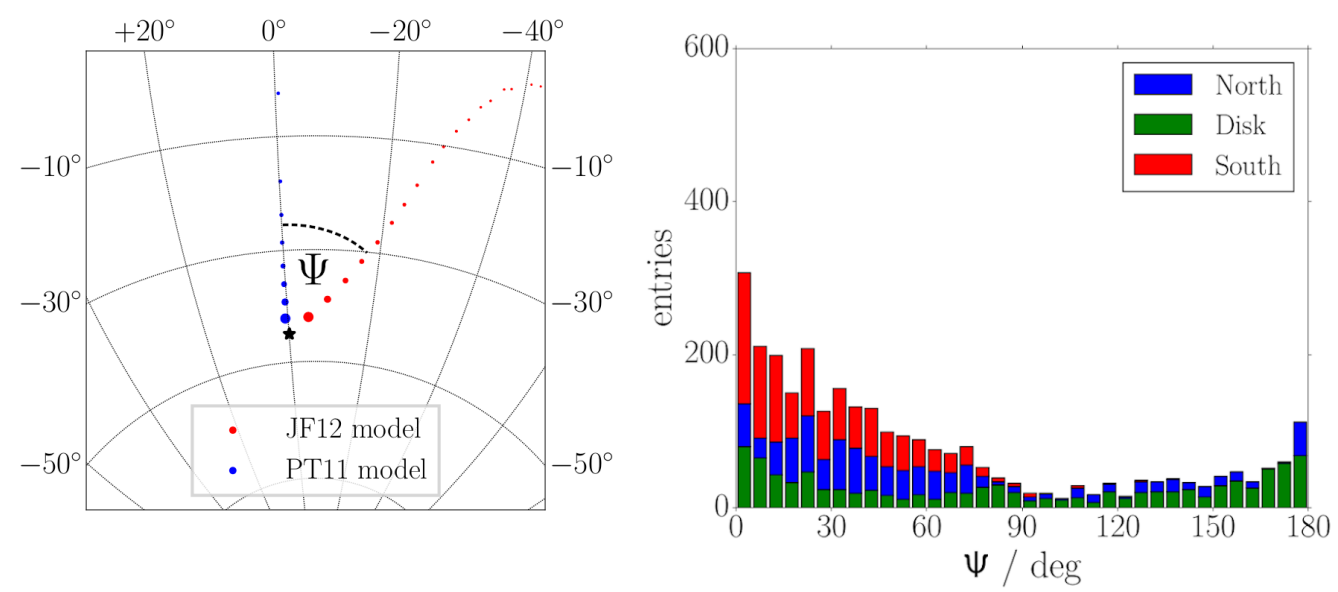

Figure 2: a) Arrival directions of UHECRs between $10^{18.5} \mathrm{eV}$ and $10^{20} \mathrm{eV}$ from a source with galactic coordinates $l=0^{\circ}$ and $b=-45^{\circ}$ after deflections in the coherent fields of the JF12 (red) and PT11 (blue) models. b) Distribution of the angular difference $\Psi$ between the JF12 and the PT11 field models for three different regions in the sky: the disk with galactic latitudes $|b|<19.5^{\circ}$ and subsequently the northern and southern sky with latitudes of $b>19.5^{\circ}$ and $b<-19.5^{\circ}$, respectively [6].

We initialize the fit method with the directional deflections as predicted by the JF12 model [3], and allow for a modification $\Psi$ (cf. figure 2a) relative to this model in the form of a differentiable function on the surface of the sphere. For this, we implement a spherical harmonics expansion and suppress variations on small angular scales by demanding the order to be at most $\ell=5$ : 


$$
\Psi(\theta, \varphi)=\sum_{\ell=0}^{5} \sum_{m=-\ell}^{\ell} a_{l}^{m} Y_{l}^{m}(\theta, \varphi) .
$$

The order $\ell=5$ yields 36 coefficients $a_{l}^{m}$ which are used as fit parameters to modify the GMF model. To adapt the modification function $\Psi(\theta, \varphi)$ to the most prominent deflection signatures we define an elliptically-shaped potential around each $\operatorname{cosmic}$ ray $i$, with the major axis aligned with the local direction of deflection as given by the JF12 model modified with the value of $\Psi_{i}:=\Psi\left(\theta_{i}, \varphi_{i}\right)$. In analogy to the principle of minimum total potential energy, all cosmic rays are interacting with each other through this potential. It follows the radial dependence of a Fisher distribution:

$$
C=-\frac{1}{N^{2}} \sum_{i} \sum_{j} \exp \left(\left(\vec{p}_{i} \cdot \vec{p}_{j}-1\right) / \sigma_{i, j}^{2}\right),
$$

where the Gaussian width is $\sigma_{\text {major }}=20^{\circ}\left(\sigma_{\text {minor }}=5^{\circ}\right)$ along the major (minor) axis. For other directions, the width is given by the radial function of an ellipse. The term $C$ is used in the objective function of the fit. It is minimal for the case that all the elliptical potentials are aligned with local elongated structures in the arrival directions.

Additionally, we add an objective term $F$, which penalizes the overall deviation from zero of the modification function $\Psi(\theta, \varphi)$ and thus the deviation from the original GMF model itself:

$$
F=\frac{1}{4 \pi} \int_{0}^{2 \pi} \int_{-\pi / 2}^{\pi / 2} \Psi^{2}(\theta, \varphi) \cos (\theta) d \theta d \varphi
$$

The total objective function exhibits a hyperparameter that we set empirically to $\lambda_{F}=2 \cdot 10^{-3}$ :

$$
J=C+\lambda_{F} \cdot F .
$$

\subsection{Second stage: Contracting alignment patterns}

In the second stage of the fit method, we obtain the estimated arrival direction $\hat{p}_{i}$ on Earth for each given pair of fit parameters $\left(\hat{s}_{i}, \hat{Z}_{i}\right)$ of the estimated source direction $\hat{s}_{i}$ and the estimated nuclear charge $\hat{Z}_{i}$ by the following mapping:

$$
\left(\begin{array}{c}
\hat{s}_{i} \\
\hat{Z}_{i}
\end{array}\right) \Longrightarrow \mathbf{R}\left(\Psi_{i}, \hat{s}_{i}\right) \cdot T_{\mathrm{JF} 12}\left(\hat{s}_{i}, \hat{Z}_{i}, E_{i}\right) \Longrightarrow \hat{p}_{i}
$$

Here, we first use the transformation prescription $T_{\mathrm{JF} 12}$ given by the JF12 GMF model to obtain preliminary arrival directions and then apply on them the GMF correction $\Psi_{i}$ in the form of a rotation $\mathbf{R}\left(\Psi_{i}, \hat{s}_{i}\right)$ around the axis $\hat{s}_{i}$ with rotation angle $\Psi_{i}$ (cf. section 2.1). In our notation, all variables with a hat refer to variables directly related to the fit.

For using the minimization technique of the TensorFlow library, the transformation prescription $T_{\mathrm{JF} 12}$ needs to be differentiable; thus, we trained a deep neural network consisting of five hidden layers with 100 fully connected nodes each to learn the projection from the input $\left(\hat{s}_{i}, \hat{Z}_{i} / E_{i}\right)$ to the output $\hat{p}_{i}$. We compare arrival directions of the simulation in the analytical JF12 field with the predictions of the neural network and find that the differentiable model is able to interpolate 
between rigidities and directions which were not used during the training process [12].

Again, we define an objective function which guides the fit parameters of equation 2.5 and consists of several terms which aim at concentrating cosmic-ray directions prior to transformation while preserving the observed quantities: the arrival directions on Earth and the shower depth maximum. In the following, the three terms of the objective function are introduced:

1) As the initial source directions $\hat{s}_{i}$ and the charges $Z_{i}$ are free fit parameters, the predicted arrival directions $\hat{p}_{i}$ have to be constrained to coincide with the measured ones:

$$
D=\frac{1}{N} \sum_{i}\left\|p_{i}-\hat{p}_{i}\right\|^{2}
$$

2) In our benchmark simulation, we have access to the shower depth maximum $X_{\max , i}$ of each cosmic ray as a charge-sensitive quantity. The shower depth is subjected to large shower to shower fluctuations and follows the asymmetric Gumbel distribution $G\left(A_{i}, E_{i}\right)$ [13]. We define $\mu_{i}:=\arg \max _{X_{\max }} G\left(\hat{A}_{i}, E_{i}\right)$ as the most probable $X_{\max }$ value and $\operatorname{Var}\left(G\left(\hat{A}_{i}, E_{i}\right)\right)$ as either the left or the right-sided width of the Gumbel distribution. Then we can constrain the cosmic rays to follow their respective Gumbel distribution by a $\chi^{2}$ formulation (detailed description in [9]):

$$
Q=\left[\frac{1}{N} \sum_{i} \frac{\left(X_{\max , i}-\mu_{i}\right)^{2}}{\operatorname{Var}\left(G\left(\hat{A}_{i}, E_{i}\right)\right)}-1\right]^{2} .
$$

3) While the previous two objective terms are designed to keep cosmic-ray quantities compatible with the measurements, the third term forces cosmic rays to originate from common sources. We use the same potential as in equation 2.2, however, with the $\Psi_{i}$ fixed by the procedure in section 2.1. The parameters of the ellipse are adapted for this application to values of $\sigma_{\text {major }}=15^{\circ}$ $\left(\sigma_{\text {minor }}=5^{\circ}\right)$ along the major (minor) axis. The shape of the potential acts on the fit by predominantly clustering the estimated source directions $\hat{s}_{i}$ along the predicted deflection of the modified GMF model.

The fit is driven by the total objective function consisting of the three terms, and the two hyperparameters are empirically determined to $\lambda_{Q}=0.1$ and $\lambda_{C}=0.5$ :

$$
J=D+\lambda_{Q} \cdot Q+\lambda_{C} \cdot C .
$$

\section{Astrophysical benchmark simulation}

To demonstrate the complete procedure of the method, we introduce a simulated astrophysical arrival scenario of UHECRs including an uncertainty in the Galactic magnetic field model.

We simulate UHECRs above energy $40 \mathrm{EeV}$ following a parametrized power-law with smooth change of the spectral index $\gamma$ which was fitted to data of the Pierre Auger Observatory [14]. The charge distribution is assumed to be energy-independent and uniform between $Z=1$ and $Z=8$, and the atmospheric depth at which the shower maximizes is randomly drawn from the energy and mass-dependent Gumbel distribution $G(E, A)$ [13], where we assume for the nuclear mass $A=2 \cdot Z$ 
and for protons $A=Z=1$. In total, we simulate 1000 cosmic-ray events, which is comparable with the recent event statistics of the Pierre Auger Observatory at this energy threshold. While 25 cosmic rays are assigned to each of four different randomly placed point-like sources in the sky, the remaining 900 cosmic rays are distributed isotropically.

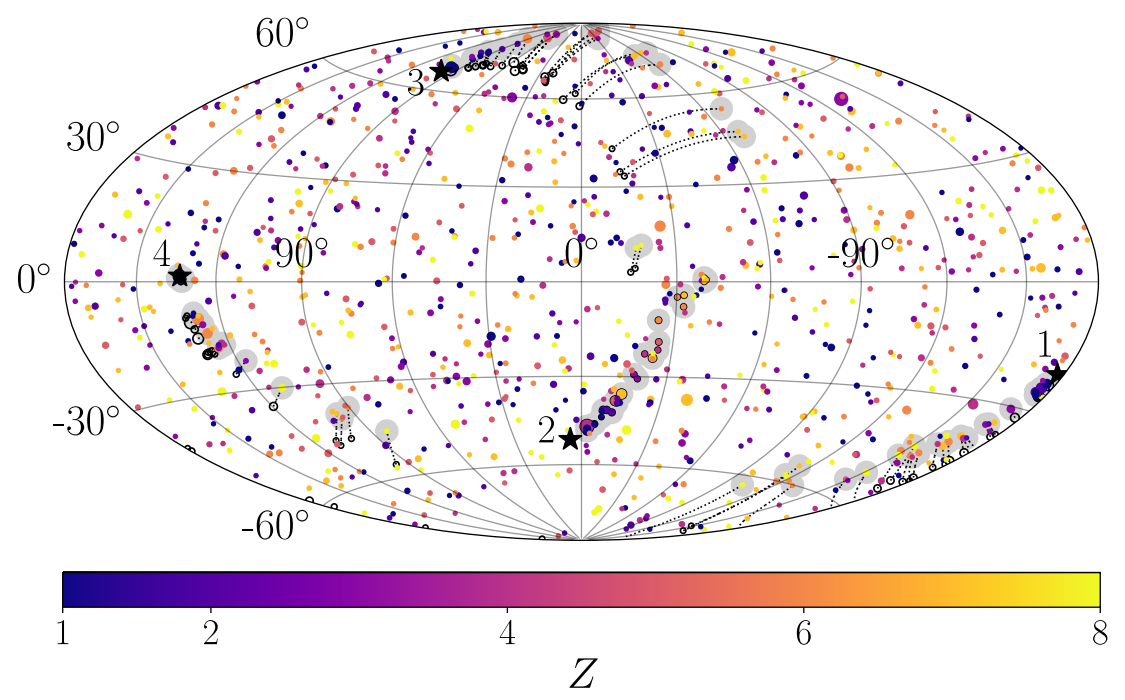

Figure 3: Demonstration of constructing the arrival scenario including the simulated uncertainty of the Galactic magnetic field. Colors denote the nuclear charge $Z$ of the cosmic ray event and gray shaded areas the cosmic rays which are assigned to one of the four sources (black stars).

The deflections in the Galactic magnetic field are simulated as follows: First, the cosmic rays with energy $E$ and charge $Z$ are propagated through the JF12 GMF model using a magnetic field lens [15]. Additionally, we apply a rigidity dependent Gaussian smearing of $\delta=0.5 \cdot Z / E[\mathrm{EeV}] \mathrm{rad}$ and visualize the arrival direction from this step by the black circular symbols in figure 3 . Now we assign to the four sources a model uncertainty in the form of the spherical angle $\Psi$ as introduced in figure $2 \mathrm{a}$, and select in good accordance with figure $2 \mathrm{~b}$ as examples $\Psi_{1}=-30^{\circ}, \Psi_{2}=0^{\circ}$, $\Psi_{3}=+45^{\circ}$ and $\Psi_{4}=+15^{\circ}$ in order of ascending galactic longitude $l$ (from right to left in figure 3). The gray shaded areas indicate the resulting arrival directions after this displacement.

\section{Modification of the Galactic magnetic field model}

We apply the method to correct the Galactic magnetic field as introduced in section 2.1 to the simulated scenario from the previous section. As can be seen in figure 4, the modification function $\Psi(\theta, \varphi)$ (cf. equation 2.1) yields average correction angles for the signal patterns (indicated by the gray symbols) of $-28^{\circ},+8^{\circ},+42^{\circ}$ and $+12^{\circ}$ for the four sources, again in order of ascending galactic longitude $l$. These values come close to the original simulated uncertainties of $-30^{\circ}, 0^{\circ}$, $+45^{\circ}$ and $+15^{\circ}$. Note, that the overall scale of the modification function $\Psi(\theta, \varphi)$ can be adapted by the hyperparameter $\lambda_{F}$ and should be set according to our confidence in the GMF model. 


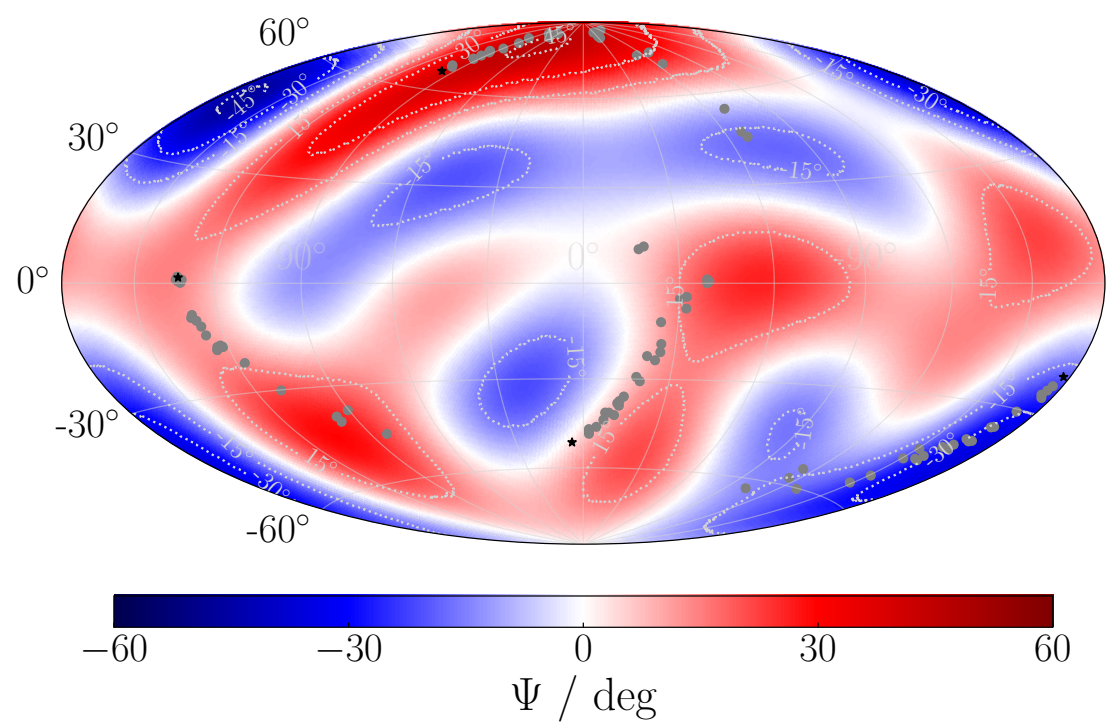

Figure 4: Fit result of the modification function $\Psi(\theta, \varphi)$ (cf. equation 2.1) for the GMF model applied to the benchmark simulation presented in figure 3. The gray circular symbols indicate the signal cosmic rays.

\section{Contracting alignment patterns}

In the second stage of the method (cf. section 2.2), the charges $\hat{Z}_{i}$ and the estimated source directions $\hat{s}_{i}$ are fitted simultaneously, while the deflections in the modified GMF model are mapping the predicted cosmic-ray fit parameters $\left(\hat{s}_{i}, \hat{Z}_{i}\right)$ to the estimated observed directions $\hat{p}_{i}$.

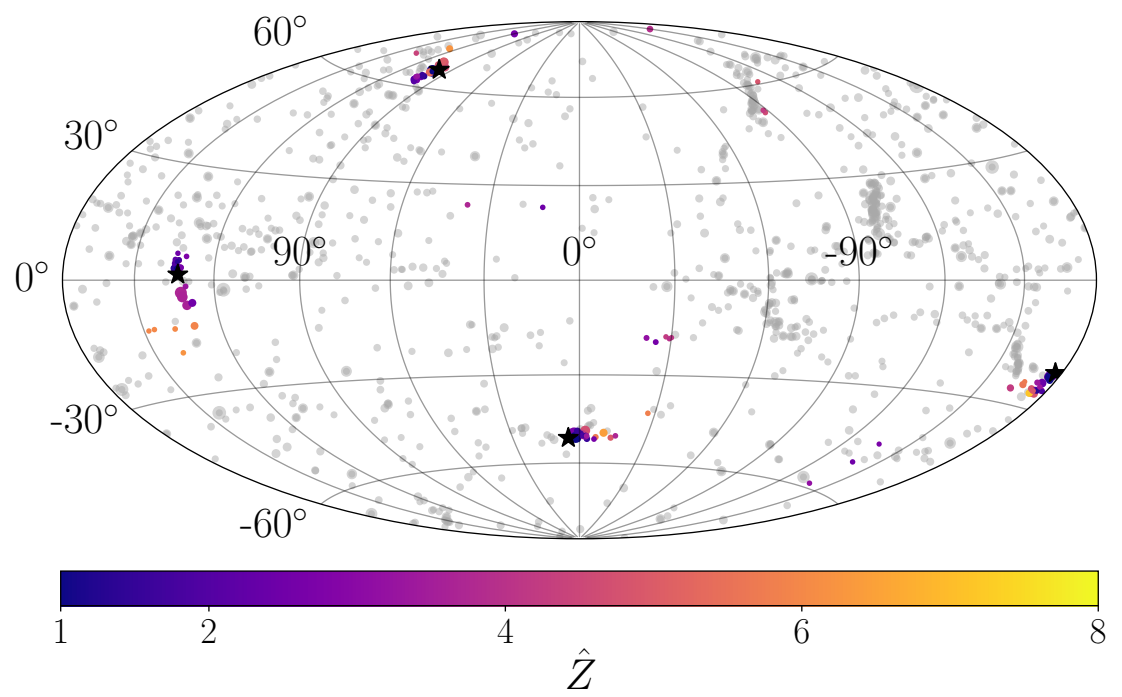

Figure 5: Skymap showing the fitted source directions $\hat{s}_{i}$ with colored symbols referring to the signal events. The color code denotes the fitted nuclear charge $\hat{Z}_{i}$ and the star symbols the original source directions.

After approximately $10^{5}$ iterations, the fit converges to values of $\left(\hat{s}_{i}, \hat{Z}_{i}\right)$ as visualized in figure 5. The majority of the signal cosmic rays (colored symbols) cluster correctly in regions close to their original source. However, also the isotropic cosmic rays (gray symbols) form clusters 
as demanded by equation 2.2 in directions which are characteristic for the Galactic magnetic field model considered. Therefore, an improvement of the relative cluster strength between signal and background cosmic rays is expected when including additional information on the backtracking of an isotropic arrival distribution through the GMF model.

To obtain the sensitivity of the found clusters, a tophat counting can be used and then compared to applications of the method on isotropic arrival distributions. While the sensitivity of the method has already been examined under the assumption that the Galactic magnetic field is completely known [9], a corresponding investigation of this work is left for future research.

\section{Conclusion}

An updated method to find mixed composition alignment patterns of ultra-high energy cosmic rays from nearby sources [9] has been presented. As a new aspect, the method initially corrects uncertainties of an existing Galactic magnetic field model by using thread-like structures in the arrival directions of cosmic rays. Only then, in a simultaneous fit to the nuclear charges and the estimated source directions of all cosmic rays, the source directions are enforced to cluster in as few directions outside the Galaxy as possible while keeping the physical properties like arrival direction and nuclear charge compatible with the measurements. In a simulated astrophysical scenario including expected uncertainties in the deflection caused by the Galactic magnetic field, we show that the method is capable of reconstructing the simulated source directions even for a faint signal in a substantial isotropic background.

\section{Acknowledgement}

This work is supported by the Ministry of Innovation, Science and Research of the State of North Rhine-Westphalia, and the Federal Ministry of Education and Research (BMBF).

\section{References}

[1] A. Aab [Pierre Auger Collaboration], Science 357 (2017) 1266

[2] A. Aab [Pierre Auger Collaboration], Astrophys. J. 853 (2018) L29

[3] R. Jansson, G. R. Farrar, The Astrophysical Journal 757 (2012) 14

[4] M. S. Pshirkov et al., The Astrophysical Journal 738 (2011) 192

[5] P. Terral, K. Ferrière, A\&A 600 (2017) A29

[6] M. Erdmann, G. Müller, M. Urban, M. Wirtz, Astropart. Phys. 85 (2016) 54-64

[7] G. Farrar, M. Unger, PoS (ICRC2017) 558

[8] A. Aab [Pierre Auger Collaboration], Phys. Rev. D 90 (2014) 122006

[9] M. Erdmann, L. Geiger, D. Schmidt, M. Urban, M. Wirtz, Astropart. Phys. 108 (2019) 74-83

[10] P. Abreu [Pierre Auger Collaboration], Astropart. Phys. 35 (2012) 354-361

[11] M. Abadi et al., 2016, arXiv:1603.04467

[12] M. Erdmann, L. Geiger, D. Schmidt, M. Urban, M. Wirtz, EPJ Web of Conferences 210 (2019) 04004

[13] M. De Domenico, M. Settimo, S. Riggi, E. Bertin, JCAP 1307 (2013) 050

[14] F. Fenu [Pierre Auger Collaboration], PoS (ICRC2017) 486, arXiv:1708.06592

[15] H.-P. Bretz, M. Erdmann, P. Schiffer, D. Walz, T. Winchen, Astropart. Phys. 54 (2014) 110 